\title{
REAL HYPERSURFACES IN COMPLEX HYPERBOLIC TWO-PLANE GRASSMANNIANS WITH COMMUTING STRUCTURE JACOBI OPERATORS
}

\author{
HYUNJIN LEE, YOUNG JIN SUH AND CHANGHWA WOO
}

\begin{abstract}
In this paper, we introduce a new commuting condition between the structure Jacobi operator and symmetric $(1,1)$-type tensor field $T$, that is, $R_{\xi} \phi T=T R_{\xi} \phi$, where $T=A$ or $T=S$ for Hopf hypersurfaces in complex hyperbolic two-plane Grassmannians. By using simultaneous diagonalzation for commuting symmetric operators, we give a complete classification of real hypersurfaces in complex hyperbolic two-plane Grassmannians with commuting condition respectively.
\end{abstract}

\section{INTRODUCTION}

It is one of the main topics in submanifold geometry to investigate immersed real hypersurfaces of homogeneous type in Hermitian symmetric spaces of rank 2 (HSS2) with certain geometric conditions. Understanding and classifying real hypersurfaces in HSS2 is one of important problems in differential geometry. One of these spaces is the complex two-plane Grassmannian $G_{2}\left(\mathbb{C}^{m+2}\right)=S U_{2+m} / S\left(U_{2} \cdot U_{m}\right)$ defined by the set of all complex two-dimensional linear subspaces in $\mathbb{C}^{m+2}$. Another one is the complex hyperbolic two-plane Grassmannian $G_{2}^{*}\left(\mathbb{C}^{m+2}\right)=S U_{2, m} / S\left(U_{2} \cdot U_{m}\right)$ defined by the set of all complex two-dimensional linear subspaces in indefinite complex Euclidean space $\mathbb{C}_{2}^{m+2}$.

These are typical examples of HSS2. Characterizing typical model spaces of real hypersurfaces under certain geometric conditions is one of our main interests in the classification theory in $G_{2}\left(\mathbb{C}^{m+2}\right)$ or $S U_{2, m} / S\left(U_{2} \cdot U_{m}\right)$ (see [13] and [14]).

Our recent interest is the study by applying geometric conditions used in submanifolds in $G_{2}\left(\mathbb{C}^{m+2}\right)$ to submanifolds in $S U_{2, m} / S\left(U_{2} \cdot U_{m}\right)$.

$G_{2}\left(\mathbb{C}^{m+2}\right)=S U_{2+m} / S\left(U_{2} \cdot U_{m}\right)$ has compact transitive group $S U_{2+m}$, however $S U_{2, m} / S\left(U_{2} \cdot U_{m}\right)$ has noncompact indefinite transitive group $S U_{2, m}$. This distinction gives various remarkable results.

The complex hyperbolic two-plane Grassmannian $S U_{2, m} / S\left(U_{2} \cdot U_{m}\right)$ is the unique noncompact, irreducible, Kähler and quaternionic Kähler manifold which is not a hyperkähler manifold.

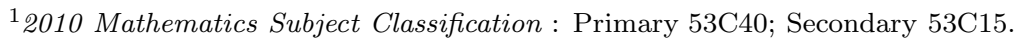

${ }^{2}$ Key words : Real hypersurfaces; complex hyperbolic two-plane Grassmannians, Hopf hypersurface, shape operator, Ricci tensor, structure Jacobi operator, commuting condition.

*This work was supported by Grant Proj. No. NRF-2015-R1A2A1A-01002459 and the third author is supported by NRF Grant funded by the Korean Government (NRF-2013-Fostering Core Leaders of Future Basic Science Program).
} 
Let $M$ be a real hypersurface in complex hyperbolic two-plane Grassmannian $S U_{2, m} / S\left(U_{2} \cdot U_{m}\right)$. Let $N$ be a local unit normal vector field on $M$. Since the complex hyperbolic two-plane Grassmannians $S U_{2, m} / S\left(U_{2} \cdot U_{m}\right)$ has the Kähler structure $J$, we may define a Reeb vector field $\xi=-J N$ and a 1-dimensional distribution $\mathcal{C}^{\perp}=\operatorname{Span}\{\xi\}$.

Let $\mathcal{C}$ be the orthogonal complement of distribution $\mathcal{C}^{\perp}$ in $T_{p} M$ at $p \in M$. It is the complex maximal subbundle of $T_{p} M$. Thus the tangent space of $M$ consists of the direct sum of $\mathcal{C}$ and $\mathcal{C}^{\perp}$ as follows: $T_{p} M=\mathcal{C} \oplus \mathcal{C}^{\perp}$. The real hypersurface $M$ is said to be Hopf if $A \mathcal{C} \subset \mathcal{C}$, or equivalently, the Reeb vector field $\xi$ is principal with principal curvature $\alpha=g(A \xi, \xi)$, where $g$ denotes the metric. In this case, the principal curvature $\alpha$ is said to be a Reeb curvature of $M$.

From the quaternionic Kähler structure $\mathfrak{J}=\operatorname{Span}\left\{J_{1}, J_{2}, J_{3}\right\}$ of $S U_{2, m} / S\left(U_{2} \cdot U_{m}\right)$, there naturally exist almost contact 3-structure vector fields $\xi_{\nu}=-J_{\nu} N, \nu=1,2,3$. Let $\mathcal{Q}^{\perp}=\operatorname{Span}\left\{\xi_{1}, \xi_{2}, \xi_{3}\right\}$. It is a 3-dimensional distribution in the tangent space $T_{p} M$ of $M$ at $p \in M$. In addition, $\mathcal{Q}$ stands for the orthogonal complement of $\mathcal{Q}^{\perp}$ in $T_{p} M$. It is the quaternionic maximal subbundle of $T_{p} M$. Thus the tangent space of $M$ can be splitted into $\mathcal{Q}$ and $\mathcal{Q}^{\perp}$ as follows: $T_{p} M=\mathcal{Q} \oplus \mathcal{Q}^{\perp}$.

Thus, we have considered two natural geometric conditions for real hypersurfaces in $S U_{2, m} / S\left(U_{2} \cdot U_{m}\right)$ such that the subbundles $\mathcal{C}$ and $\mathcal{Q}$ of $T M$ are both invariant under the shape operator. By using these geometric conditions, we will use the results in Suh [13, Theorem 1].

On the other hand, a Jacobi field along geodesics of a given Riemannian manifold $(\bar{M}, \bar{g})$ plays an important role in the study of differential geometry. It satisfies a well-known differential equation which inspires Jacobi operators. It is defined by $\left(\bar{R}_{X}(Y)\right)(p)=(\bar{R}(Y, X) X)(p)$, where $\bar{R}$ denotes the curvature tensor of $\bar{M}$ and $X, Y$ denote any vector fields on $\bar{M}$. It is known to be a self-adjoint endomorphism on the tangent space $T_{p} \bar{M}, p \in \bar{M}$. Clearly, each tangent vector field $X$ to $\bar{M}$ provides a Jacobi operator with respect to $X$. Thus the Jacobi operator on a real hypersurface $M$ of $\bar{M}$ with respect to $\xi$ is said to be a structure Jacobi operator and will be denoted by $R_{\xi}$. The Riemannian curvature tensor of $M$ (resp., $\bar{M}$ ) is denoted by $R$ (resp., $\bar{R}$ ).

For a commuting problem concerned with the structure Jacobi operator $R_{\xi}$ and the structure tensor $\phi$ of Hopf hypersurface $M$ in $G_{2}\left(\mathbb{C}^{m+2}\right)$, that is, $R_{\xi} \phi A=A R_{\xi} \phi$, Lee, Suh and Woo [3] proved that a Hopf hypersurface $M$ with $R_{\xi} \phi A=A R_{\xi} \phi$ and $\xi \alpha=0$ is locally congruent to an open part of a tube around a totally geodesic $G_{2}\left(\mathbb{C}^{m+1}\right)$ in $G_{2}\left(\mathbb{C}^{m+2}\right)$. Motivated by this result, we consider the same condition in the different ambient space, that is,

$$
R_{\xi} \phi A X=A R_{\xi} \phi X
$$

for any tangent vector field $X$ on $M$ in $S U_{2, m} / S\left(U_{2} \cdot U_{m}\right)$. The geometric meaning of $R_{\xi} \phi A X=A R_{\xi} \phi X$ can be explained in such a way that any eigenspace of $R_{\xi}$ on the distribution $\mathcal{C}=\left\{X \in T_{p} M \mid X \perp \xi\right\}, p \in M$, is invariant under the shape operator $A$ of $M$ in $S U_{2, m} / S\left(U_{2} \cdot U_{m}\right)$. Then by using [13, Theorem 1], we give a complete classification of Hopf hypersurfaces in $S U_{2, m} / S\left(U_{2} \cdot U_{m}\right)$ with $R_{\xi} \phi A X=A R_{\xi} \phi X$ as follows:

Theorem 1. Let $M$ be a Hopf hypersurface in complex hyperbolic two-plane Grassmannians $S U_{2, m} / S\left(U_{2} \cdot U_{m}\right), m \geq 3$ with $R_{\xi} \phi A=A R_{\xi} \phi$. If the Reeb curvature 
$\alpha=g(A \xi, \xi)$ is constant along the Reeb direction of the structure vector field $\xi$, then $M$ is locally congruent to one of the following:

(i) a tube over a totally geodesic $S U_{2, m-1} / S\left(U_{2} \cdot U_{m-1}\right)$ in $S U_{2, m} / S\left(U_{2} \cdot U_{m}\right)$ or

(ii) a horosphere in $S U_{2, m} / S\left(U_{2} \cdot U_{m}\right)$ whose center at infinity is singular and of type $J X \in \mathfrak{J} X$.

From the Riemannian curvature tensor $R$ of $M$ in $S U_{2, m} / S\left(U_{2} \cdot U_{m}\right)$ we can define the Ricci tensor $S$ of $M$ in such a way that

$$
g(S X, Y)=\sum_{i=1}^{4 m-1} g\left(R\left(e_{i}, X\right) Y, e_{i}\right),
$$

where $\left\{e_{1}, \cdots, e_{4 m-1}\right\}$ denotes a basis of the tangent space $T_{p} M$ of $M, p \in M$, in $S U_{2, m} / S\left(U_{2} \cdot U_{m}\right)$ (see [15]). Then we can consider another new commuting condition

$$
R_{\xi} \phi S X=S R_{\xi} \phi X
$$

for any tangent vector field $X$ on $M$. That is, the operator $R_{\xi} \phi$ commutes with the Ricci tensor $S$.

Then by [13, Theorem 1], we also give another classification related to the Ricci tensor $S$ of $M$ in $S U_{2, m} / S\left(U_{2} \cdot U_{m}\right)$ as follows:

Theorem 2. Let $M$ be a Hopf hypersurface in complex hyperbolic two-plane Grassmannians $S U_{2, m} / S\left(U_{2} \cdot U_{m}\right), m \geq 3$ with $R_{\xi} \phi S=S R_{\xi} \phi$. If the smooth function $\alpha=g(A \xi, \xi)$ is constant along the direction of $\xi$, then $M$ is locally congruent to one of the following:

(i) a tube over a totally geodesic $S U_{2, m-1} / S\left(U_{2} \cdot U_{m-1}\right)$ in $S U_{2, m} / S\left(U_{2} \cdot U_{m}\right)$ or

(ii) a horosphere in $S U_{2, m} / S\left(U_{2} \cdot U_{m}\right)$ whose center at infinity is singular and of type $J X \in \mathfrak{J} X$.

In this paper, we refer [10, 13, 14, and 15] for Riemannian geometric structures of complex hyperboilc two-plane Grassmannians $S U_{2, m} / S\left(U_{2} \cdot U_{m}\right), m \geq 3$.

\section{The Complex hyperbolic two-Plane Grassmannian $S U_{2, m} / S\left(U_{2} \cdot U_{m}\right)$}

In this section we summarize basic material about complex hyperbolic two-plane Grassmann manifolds $S U_{2, m} / S\left(U_{2} \cdot U_{m}\right)$, for details we refer to [9, 11, [13] and [15. The Riemannian symmetric space $S U_{2, m} / S\left(U_{2} \cdot U_{m}\right)$, which consists of all complex two-dimensional linear subspaces in indefinite complex Euclidean space $\mathbb{C}_{2}^{m+2}$ is a connected, simply connected, irreducible Riemannian symmetric space of noncompact type and with rank two. Let $G=S U_{2, m}$ and $K=S\left(U_{2} \cdot U_{m}\right)$, and denote by $\mathfrak{g}$ and $\mathfrak{k}$ the corresponding Lie algebra of the Lie group $G$ and $K$ respectively. Let $B$ be the Killing form of $\mathfrak{g}$ and denote by $\mathfrak{p}$ the orthogonal complement of $\mathfrak{k}$ in $\mathfrak{g}$ with respect to $B$. The resulting decomposition $\mathfrak{g}=\mathfrak{k} \oplus \mathfrak{p}$ is a Cartan decomposition of $\mathfrak{g}$. The Cartan involution $\theta \in \mathrm{A} u t(\mathfrak{g})$ on $\mathfrak{s u}_{2, m}$ is given by $\theta(A)=I_{2, m} A I_{2, m}$, where

$$
I_{2, m}=\left(\begin{array}{cc}
-I_{2} & 0_{2, m} \\
0_{m, 2} & I_{m}
\end{array}\right),
$$

$I_{2}$ and $I_{m}$ denote the identity $2 \times 2$-matrix and $m \times m$-matrix respectively. Then $<X, Y>=-B(X, \theta Y)$ becomes a positive definite $\mathrm{A} d(K)$-invariant inner product 
on $\mathfrak{g}$. Its restriction to $\mathfrak{p}$ induces a metric $g$ on $S U_{2, m} / S\left(U_{2} \cdot U_{m}\right)$, which is also known as the Killing metric on $S U_{2, m} / S\left(U_{2} \cdot U_{m}\right)$. Throughout this paper we consider $S U_{2, m} / S\left(U_{2} \cdot U_{m}\right)$ together with this particular Riemannian metric $g$.

The Lie algebra $\mathfrak{k}$ decomposes orthogonally into $\mathfrak{k}=\mathfrak{s u}_{2} \oplus \mathfrak{s} \mathfrak{u}_{m} \oplus \mathfrak{u}_{1}$, where $\mathfrak{u}_{1}$ is the one-dimensional center of $\mathfrak{k}$. The adjoint action of $\mathfrak{s u}_{2}$ on $\mathfrak{p}$ induces the quaternionic Kähler structure $\mathfrak{J}$ on $S U_{2, m} / S\left(U_{2} \cdot U_{m}\right)$, and the adjoint action of

$$
Z=\left(\begin{array}{cc}
\frac{m i}{m+2} I_{2} & 0_{2, m} \\
0_{m, 2} & \frac{-2 i}{m+2} I_{m}
\end{array}\right) \in \mathfrak{u}_{1}
$$

induces the Kähler structure $J$ on $S U_{2, m} / S\left(U_{2} \cdot U_{m}\right)$. By construction, $J$ commutes with each almost Hermitian structure $J_{\nu}$ in $\mathfrak{J}$ for $\nu=1,2,3$. Recall that a canonical local basis $\left\{J_{1}, J_{2}, J_{3}\right\}$ of a quaternionic Kähler structure $\mathfrak{J}$ consists of three almost Hermitian structures $J_{1}, J_{2}, J_{3}$ in $\mathfrak{J}$ such that $J_{\nu} J_{\nu+1}=J_{\nu+2}=-J_{\nu+1} J_{\nu}$, where the index $\nu$ is to be taken modulo 3. The tensor field $J J_{\nu}$, which is locally defined on $S U_{2, m} / S\left(U_{2} \cdot U_{m}\right)$, is self-adjoint and satisfies $\left(J J_{\nu}\right)^{2}=I$ and $\operatorname{tr}\left(J J_{\nu}\right)=0$, where $I$ is the identity transformation. For a nonzero tangent vector $X$, we define $\mathbb{R} X=\{\lambda X \mid \lambda \in \mathbb{R}\}, \mathbb{C} X=\mathbb{R} X \oplus \mathbb{R} J X$, and $\mathbb{H} X=\mathbb{R} X \oplus \mathfrak{J} X$.

We identify the tangent space $T_{o} S U_{2, m} / S\left(U_{2} \cdot U_{m}\right)$ of $S U_{2, m} / S\left(U_{2} \cdot U_{m}\right)$ at $o$ with $\mathfrak{p}$ in the usual way. Let $\mathfrak{a}$ be a maximal abelian subspace of $\mathfrak{p}$. Since $S U_{2, m} / S\left(U_{2} \cdot U_{m}\right)$ has rank two, the dimension of any such subspace is two. Every nonzero tangent vector $X \in T_{o} S U_{2, m} / S\left(U_{2} \cdot U_{m}\right) \cong \mathfrak{p}$ is contained in some maximal abelian subspace of $\mathfrak{p}$. Generically this subspace is uniquely determined by $X$, in which case $X$ is called regular. If there exist more than one maximal abelian subspaces of $\mathfrak{p}$ containing $X$, then $X$ is called singular. There is a simple and useful characterization of the singular tangent vectors: A nonzero tangent vector $X \in \mathfrak{p}$ is singular if and only if $J X \in \mathfrak{J} X$ or $J X \perp \mathfrak{J} X$.

Up to scaling there exists a unique $S U_{2, m}$-invariant Riemannian metric $g$ on $S U_{2, m} / S\left(U_{2} \cdot U_{m}\right)$. Equipped with this metric, $S U_{2, m} / S\left(U_{2} \cdot U_{m}\right)$ is a Riemannian symmetric space of rank two which is both Kähler and quaternionic Kähler. For computational reasons we normalize $g$ such that the minimal sectional curvature of $\left(S U_{2, m} / S\left(U_{2} \cdot U_{m}\right), g\right)$ is -4 . The sectional curvature $K$ of the noncompact symmetric space $S U_{2, m} / S\left(U_{2} \cdot U_{m}\right)$ equipped with the Killing metric $g$ is bounded by $-4 \leq K \leq 0$. The sectional curvature -4 is obtained for all two-planes $\mathbb{C} X$ when $X$ is a non-zero vector with $J X \in \mathfrak{J} X$.

When $m=1, G_{2}^{*}\left(\mathbb{C}^{3}\right)=S U_{1,2} / S\left(U_{1} \cdot U_{2}\right)$ is isometric to the two-dimensional complex hyperbolic space $\mathbb{C} H^{2}$ with constant holomorphic sectional curvature -4 .

When $m=2$, we note that the isomorphism $S O(4,2) \simeq S U_{2,2}$ yields an isometry between $G_{2}^{*}\left(\mathbb{C}^{4}\right)=S U_{2,2} / S\left(U_{2} \cdot U_{2}\right)$ and the indefinite real Grassmann manifold $G_{2}^{*}\left(\mathbb{R}_{2}^{6}\right)$ of oriented two-dimensional linear subspaces of an indefinite Euclidean space $\mathbb{R}_{2}^{6}$. For this reason we assume $m \geq 3$ from now on, although many of the subsequent results also hold for $m=1,2$.

From now on, hereafter $X, Y$ and $Z$ always stand for any tangent vector fields on $M$. 
The Riemannian curvature tensor $\bar{R}$ of $S U_{2, m} / S\left(U_{2} \cdot U_{m}\right)$ is locally given by

$$
\begin{aligned}
-2 \bar{R}(X, Y) Z=g(Y, Z) & X-g(X, Z) Y+g(J Y, Z) J X \\
& -g(J X, Z) J Y-2 g(J X, Y) J Z \\
& +\sum_{\nu=1}^{3}\left\{g\left(J_{\nu} Y, Z\right) J_{\nu} X-g\left(J_{\nu} X, Z\right) J_{\nu} Y-2 g\left(J_{\nu} X, Y\right) J_{\nu} Z\right\} \\
& +\sum_{\nu=1}^{3}\left\{g\left(J_{\nu} J Y, Z\right) J_{\nu} J X-g\left(J_{\nu} J X, Z\right) J_{\nu} J Y\right\},
\end{aligned}
$$

where $\left\{J_{1}, J_{2}, J_{3}\right\}$ is any canonical local basis of $\mathfrak{J}$.

\section{Fundamental FORMUlas IN $S U_{2, m} / S\left(U_{2} \cdot U_{m}\right)$}

In this section, we derive some basic formulas and the Codazzi equation for a real hypersurface in $S U_{2, m} / S\left(U_{2} \cdot U_{m}\right)$ (see [13, [14] and [15]).

Let $M$ be a real hypersurface in complex hyperbolic two-plane Grassmannian $S U_{2, m} / S\left(U_{2} \cdot U_{m}\right)$, that is, a hypersurface in $S U_{2, m} / S\left(U_{2} \cdot U_{m}\right)$ with real codimension one. The induced Riemannian metric on $M$ will also be denoted by $g$, and $\nabla$ denotes the Levi Civita covariant derivative of $(M, g)$. We denote by $\mathcal{C}$ and $\mathcal{Q}$ the maximal complex and quaternionic subbundle of the tangent bundle $T M$ of $M$, respectively. Now let us put

$$
J X=\phi X+\eta(X) N, \quad J_{\nu} X=\phi_{\nu} X+\eta_{\nu}(X) N
$$

for any tangent vector field $X$ of a real hypersurface $M$ in $S U_{2, m} / S\left(U_{2} \cdot U_{m}\right)$, where $\phi X$ denotes the tangential component of $J X$ and $N$ a unit normal vector field of $M$ in $S U_{2, m} / S\left(U_{2} \cdot U_{m}\right)$.

From the Kähler structure $J$ of $S U_{2, m} / S\left(U_{2} \cdot U_{m}\right)$ there exists an almost contact metric structure $(\phi, \xi, \eta, g)$ induced on $M$ in such a way that

$$
\phi^{2} X=-X+\eta(X) \xi, \quad \eta(\xi)=1, \quad \phi \xi=0, \quad \eta(X)=g(X, \xi)
$$

for any vector field $X$ on $M$. Furthermore, let $\left\{J_{1}, J_{2}, J_{3}\right\}$ be a canonical local basis of $\mathfrak{J}$. Then the quaternionic Kähler structure $J_{\nu}$ of $S U_{2, m} / S\left(U_{2} \cdot U_{m}\right)$, together with the condition $J_{\nu} J_{\nu+1}=J_{\nu+2}=-J_{\nu+1} J_{\nu}$ in section 1, induces an almost contact metric 3-structure $\left(\phi_{\nu}, \xi_{\nu}, \eta_{\nu}, g\right)$ on $M$ as follows:

$$
\begin{aligned}
& \phi_{\nu}^{2} X=-X+\eta_{\nu}(X) \xi_{\nu}, \quad \eta_{\nu}\left(\xi_{\nu}\right)=1, \quad \phi_{\nu} \xi_{\nu}=0 \\
& \phi_{\nu+1} \xi_{\nu}=-\xi_{\nu+2}, \quad \phi_{\nu} \xi_{\nu+1}=\xi_{\nu+2}, \\
& \phi_{\nu} \phi_{\nu+1} X=\phi_{\nu+2} X+\eta_{\nu+1}(X) \xi_{\nu} \\
& \phi_{\nu+1} \phi_{\nu} X=-\phi_{\nu+2} X+\eta_{\nu}(X) \xi_{\nu+1}
\end{aligned}
$$

for any vector field $X$ tangent to $M$. Moreover, from the commuting property of $J_{\nu} J=J J_{\nu}, \nu=1,2,3$ in section 1 and (2.1), the relation between these two contact metric structures $(\phi, \xi, \eta, g)$ and $\left(\phi_{\nu}, \xi_{\nu}, \eta_{\nu}, g\right), \nu=1,2,3$, can be given by

$$
\begin{aligned}
& \phi \phi_{\nu} X=\phi_{\nu} \phi X+\eta_{\nu}(X) \xi-\eta(X) \xi_{\nu}, \\
& \eta_{\nu}(\phi X)=\eta\left(\phi_{\nu} X\right), \quad \phi \xi_{\nu}=\phi_{\nu} \xi .
\end{aligned}
$$


On the other hand, from the parallelism of Kähler structure $J$, that is, $\widetilde{\nabla} J=0$ and the quaternionic Kähler structure $\mathfrak{J}$, together with Gauss and Weingarten formulas, it follows that

$$
\begin{gathered}
\left(\nabla_{X} \phi\right) Y=\eta(Y) A X-g(A X, Y) \xi, \quad \nabla_{X} \xi=\phi A X \\
\nabla_{X} \xi_{\nu}=q_{\nu+2}(X) \xi_{\nu+1}-q_{\nu+1}(X) \xi_{\nu+2}+\phi_{\nu} A X \\
\left(\nabla_{X} \phi_{\nu}\right) Y=-q_{\nu+1}(X) \phi_{\nu+2} Y+q_{\nu+2}(X) \phi_{\nu+1} Y+\eta_{\nu}(Y) A X \\
-g(A X, Y) \xi_{\nu} .
\end{gathered}
$$

Combining these formulas, we find the following:

$$
\begin{aligned}
\nabla_{X}\left(\phi_{\nu} \xi\right)= & \nabla_{X}\left(\phi \xi_{\nu}\right) \\
= & \left(\nabla_{X} \phi\right) \xi_{\nu}+\phi\left(\nabla_{X} \xi_{\nu}\right) \\
= & q_{\nu+2}(X) \phi_{\nu+1} \xi-q_{\nu+1}(X) \phi_{\nu+2} \xi+\phi_{\nu} \phi A X \\
& -g(A X, \xi) \xi_{\nu}+\eta\left(\xi_{\nu}\right) A X .
\end{aligned}
$$

Finally, using the explicit expression for the Riemannian curvature tensor $\bar{R}$ of $S U_{2, m} / S\left(U_{2} \cdot U_{m}\right)$ in 14, the Codazzi equation takes the form

$$
\begin{aligned}
-2\left(\nabla_{X} A\right) Y+2( & \left.\nabla_{Y} A\right) X=\eta(X) \phi Y-\eta(Y) \phi X-2 g(\phi X, Y) \xi \\
& +\sum_{\nu=1}^{3}\left\{\eta_{\nu}(X) \phi_{\nu} Y-\eta_{\nu}(Y) \phi_{\nu} X-2 g\left(\phi_{\nu} X, Y\right) \xi_{\nu}\right\} \\
& +\sum_{\nu=1}^{3}\left\{\eta_{\nu}(\phi X) \phi_{\nu} \phi Y-\eta_{\nu}(\phi Y) \phi_{\nu} \phi X\right\} \\
& +\sum_{\nu=1}^{3}\left\{\eta(X) \eta_{\nu}(\phi Y)-\eta(Y) \eta_{\nu}(\phi X)\right\} \xi_{\nu},
\end{aligned}
$$

for any vector fields $X$ and $Y$ on $M$.

On the other hand, by differentiating $A \xi=\alpha \xi$ and using (2.9), we get the following

$$
\begin{aligned}
& g(\phi X, Y)-\sum_{\nu=1}^{3}\left\{\eta_{\nu}(X) \eta_{\nu}(\phi Y)-\eta_{\nu}(Y) \eta_{\nu}(\phi X)-g\left(\phi_{\nu} X, Y\right) \eta_{\nu}(\xi)\right\} \\
& \quad=g\left(\left(\nabla_{X} A\right) Y-\left(\nabla_{Y} A\right) X, \xi\right) \\
& \quad=g\left(\left(\nabla_{X} A\right) \xi, Y\right)-g\left(\left(\nabla_{Y} A\right) \xi, X\right) \\
& \quad=(X \alpha) \eta(Y)-(Y \alpha) \eta(X)+\alpha g((A \phi+\phi A) X, Y)-2 g(A \phi A X, Y) .
\end{aligned}
$$

Putting $X=\xi$ gives

$$
Y \alpha=(\xi \alpha) \eta(Y)+2 \sum_{\nu=1}^{3} \eta_{\nu}(\xi) \eta_{\nu}(\phi Y) .
$$

Then, substituting (2.11) into (2.10) the above equation, we have the following

$$
\begin{aligned}
A \phi A Y= & \frac{\alpha}{2}(A \phi+\phi A) Y+\sum_{\nu=1}^{3}\left\{\eta(Y) \eta_{\nu}(\xi) \phi \xi_{\nu}+\eta_{\nu}(\xi) \eta_{\nu}(\phi Y) \xi\right\} \\
& -\frac{1}{2} \phi Y-\frac{1}{2} \sum_{\nu=1}^{3}\left\{\eta_{\nu}(Y) \phi \xi_{\nu}+\eta_{\nu}(\phi Y) \xi_{\nu}+\eta_{\nu}(\xi) \phi_{\nu} Y\right\} .
\end{aligned}
$$


By differentiating and using (2.4), (2.5) and (2.6), we have

$$
\begin{aligned}
\nabla_{X}(\operatorname{grad} \alpha)= & X(\xi \alpha) \xi+(\xi \alpha) \phi A X \\
& -2 \sum_{\nu=1}^{3}\left\{q_{\nu+2}(X) \eta_{\nu+1}(\xi)-q_{\nu+1}(X) \eta_{\nu+2}(\xi)+2 \eta_{\nu}(\phi A X)\right\} \phi \xi_{\nu} \\
& -2 \sum_{\nu=1}^{3} \eta_{\nu}(\xi)\left\{-q_{\nu+1}(X) \phi_{\nu+2} \xi+q_{\nu+2}(X) \phi_{\nu+1} \xi+\eta_{\nu}(\xi) A X\right. \\
& \left.-g(A X, \xi) \xi_{\nu}+\phi_{\nu} \phi A X\right\} \\
= & X(\xi \alpha) \xi+(\xi \alpha) \phi A X-4 \sum_{\nu=1}^{3} \eta_{\nu}(\phi A X) \phi \xi_{\nu} \\
& -2 \sum_{\nu=1}^{3} \eta_{\nu}(\xi)\left\{\eta_{\nu}(\xi) A X-g(A X, \xi) \xi_{\nu}+\phi_{\nu} \phi A X\right\} .
\end{aligned}
$$

By taking the skew-symmetric part to the above equation, we have

$$
\begin{aligned}
0= & X(\xi \alpha) \eta(Y)-Y(\xi \alpha) \eta(X)+(\xi \alpha) g((A \phi+\phi A) X, Y) \\
& -4 \sum_{\nu=1}^{3}\left\{\eta_{\nu}(\phi A X) g\left(\phi \xi_{\nu}, Y\right)-\eta_{\nu}(\phi A Y) g\left(\phi \xi_{\nu}, X\right)\right\} \\
& +2 \alpha \sum_{\nu=1}^{3} \eta_{\nu}(\xi)\left\{\eta(X) \eta_{\nu}(Y)-\eta(Y) \eta_{\nu}(X)\right\} \\
& -2 \sum_{\nu=1}^{3} \eta_{\nu}(\xi)\left\{g\left(\phi_{\nu} \phi A X, Y\right)-g\left(\phi_{\nu} \phi A Y, X\right)\right\} .
\end{aligned}
$$

From this, by putting $X=\xi$ we have the following

$$
Y(\xi \alpha)=\xi(\xi \alpha) \eta(Y)+2 \alpha \sum_{\nu=1}^{3} \eta_{\nu}(\xi) \eta_{\nu}(Y)-2 \sum_{\nu=1}^{3} \eta_{\nu}(\xi) \eta_{\nu}(A Y) .
$$

From this, if we assume that $\xi \alpha=0$, then it follows that

$$
\sum_{\nu=1}^{3} \eta_{\nu}(\xi) \eta_{\nu}(A X)=\alpha \sum_{\nu=1}^{3} \eta_{\nu}(\xi) \eta_{\nu}(X) .
$$

Lemma 2.1. Let $M$ be a Hopf real hypersurface in $S U_{2, m} / S\left(U_{2} \cdot U_{m}\right)$. If the principal curvature $\alpha$ is constant along the direction of $\xi$, then the distribution $\mathcal{Q}$ or $\mathcal{Q}^{\perp}$ component of the structure vector field $\xi$ is invariant by the shape operator.

\section{Proof of TheOrem 1}

Let $M$ be a Hopf hypersurface in $S U_{2, m} / S\left(U_{2} \cdot U_{m}\right)$ with

$$
R_{\xi} \phi A X=A R_{\xi} \phi X
$$

The structure Jacobi operator $R_{\xi}$ of $M$ is defined by $R_{\xi} X=R(X, \xi) \xi$ for any tangent vector $X \in T_{p} M, p \in M$ (see [1] and [7]). Then for any tangent vector 
field $X$ on $M$ in $S U_{2, m} / S\left(U_{2} \cdot U_{m}\right)$, we calculate the structure Jacobi operator $R_{\xi}$

$$
\begin{aligned}
2 R_{\xi}(X)= & 2 R(X, \xi) \xi \\
= & -X+\eta(X) \xi+\sum_{\nu=1}^{3}\left\{\eta_{\nu}(X) \xi_{\nu}-\eta(X) \eta_{\nu}(\xi) \xi_{\nu}\right. \\
& \left.+3 \eta_{\nu}(\phi X) \phi_{\nu} \xi+\eta_{\nu}(\xi) \phi_{\nu} \phi X\right\}+2 \alpha A X-2 \eta(A X) A \xi,
\end{aligned}
$$

where $\alpha$ denotes the Reeb curvature defined by $g(A \xi, \xi)$.

Lemma 3.1. Let $M$ be a Hopf hypersurface in $S U_{2, m} / S\left(U_{2} \cdot U_{m}\right)$ with the commuting condition $R_{\xi} \phi A X=A R_{\xi} \phi X$. If the smooth function $\alpha$ is constant along the direction of $\xi$ on $M$, then the Reeb vector field $\xi$ belongs to either the distribution $\mathcal{Q}$ or the distribution $\mathcal{Q}^{\perp}$.

Proof. To prove this lemma, without loss of generality, $\xi$ may be written as

$$
\xi=\eta\left(X_{0}\right) X_{0}+\eta\left(\xi_{1}\right) \xi_{1}
$$

where $X_{0}$ (resp., $\xi_{1}$ ) is a unit vector in $\mathcal{Q}$ (resp., $\mathcal{Q}^{\perp}$ ) and $\eta\left(X_{0}\right) \eta\left(\xi_{1}\right) \neq 0$.

From (*) and $\phi \xi=0$, we have

$$
\left\{\begin{array}{l}
\phi X_{0}=-\eta\left(\xi_{1}\right) \phi_{1} X_{0}, \\
\phi \xi_{1}=\phi_{1} \xi=\eta\left(X_{0}\right) \phi_{1} X_{0}, \\
\phi_{1} \phi X_{0}=\eta_{1}(\xi) X_{0} .
\end{array}\right.
$$

Let $\mathfrak{U}=\{p \in M \mid \alpha(p) \neq 0\}$ be an open subset of $M$. From now on, we discuss our arguments on $\mathfrak{U}$. By virtue of Lemma 2.1] $\xi \alpha=0$ gives $A X_{0}=\alpha X_{0}$ and $A \xi_{1}=\alpha \xi_{1}$. The equation (2.12) yields $\alpha A \phi X_{0}=\left(\alpha^{2}-2 \eta^{2}\left(X_{0}\right)\right) \phi X_{0}$ by substituting $X=X_{0}$. Since $\alpha$ is non-vanishing on $\mathfrak{U}$, it becomes

$$
A \phi X_{0}=\sigma \phi X_{0}
$$

where $\sigma=\frac{\alpha^{2}-2 \eta^{2}\left(X_{0}\right)}{\alpha}$.

From (3.2) and (3.3), we have

$$
\left\{\begin{array}{l}
R_{\xi}\left(X_{0}\right)=\alpha^{2} X_{0}-\alpha^{2} \eta\left(X_{0}\right) \xi, \\
R_{\xi}\left(\xi_{1}\right)=\alpha^{2} \xi_{1}-\alpha^{2} \eta\left(\xi_{1}\right) \xi, \\
R_{\xi}\left(\phi X_{0}\right)=\left(\alpha^{2}-4 \eta^{2}\left(X_{0}\right)\right) \phi X_{0} .
\end{array}\right.
$$

On $\mathfrak{U}$, substituting $X$ by $\phi X_{0}$ into (C-1), we have

$$
X_{0}-\eta\left(X_{0}\right) \xi=0,
$$

which is a contradiction. Therefore, $\mathfrak{U}=\emptyset$, and thus it must be $p \in M-\mathfrak{U}$. Since the set $M-\mathfrak{U}=\operatorname{Int}(M-\mathfrak{U}) \cup \partial(M-\mathfrak{U})$, we consider the following two cases. Here Int (resp., $\partial$ ) denotes an interior (resp., the boundary) of $(M-\mathfrak{U})$.

- Case 1. $p \in \operatorname{Int}(M-\mathfrak{U})$.

If $p \in \operatorname{Int}(M-\mathfrak{U})$, then $\alpha=0$. For this case, it was proved by the equation (2.11).

- Case 2. $p \in \partial(M-\mathfrak{U})$.

Since $p \in \partial M-\mathfrak{U}$, there exists a sequence of points $p_{n}$ such that $p_{n} \rightarrow p$ with $\alpha(p)=0$ and $\alpha\left(p_{n}\right) \neq 0$. Such a sequence will have an infinite subsequence where $\eta\left(\xi_{1}\right)=0$ (in which case $\xi \in \mathcal{Q}$ at $p$, by the continuity) or an infinite subsequence where $\eta\left(X_{0}\right)=0$ (in which case $\xi \in \mathcal{Q}^{\perp}$ at $p$ ). 
Accordingly, we get a complete proof of our lemma.

From Lemma 3.1, we consider the case that $\xi$ belongs to the distribution $\mathcal{Q}^{\perp}$. Thus without loss of generality, we may put $\xi=\xi_{1}$. Differentiating $\xi=\xi_{1}$ along any direction $X \in T M$ and using (2.5) and (2.6), it gives us

$$
2 \eta_{3}(A X) \xi_{2}-2 \eta_{2}(A X) \xi_{3}+\phi_{1} A X-\phi A X=0 .
$$

Then, by using the symmetric (resp., skew-symmetric) property of the shape operator $A$ (resp., the structure tensor field $\phi$ ), we also obtain

$$
2 \eta_{3}(X) A \xi_{2}-2 \eta_{2}(X) A \xi_{3}+A \phi_{1} X-A \phi X=0 .
$$

Applying $\phi_{1}$ to (3.6), it implies

$$
2 \eta_{3}(A X) \xi_{3}+2 \eta_{2}(A X) \xi_{2}-A X+\alpha \eta(X) \xi-\phi_{1} \phi A X=0 .
$$

On the other hand, replacing $X=\phi X$ into (3.6), we have

$$
-2 \eta_{2}(X) A \xi_{2}-2 \eta_{3}(X) A \xi_{3}+A \phi_{1} \phi X-A X-\alpha \eta(X) \xi=0 .
$$

Lemma 3.2. Let $M$ be a Hopf hypersurface in $S U_{2, m} / S\left(U_{2} \cdot U_{m}\right), m \geq 3$, with $R_{\xi} \phi A=A R_{\xi} \phi$. If the Reeb vector field $\xi$ belongs to the distribution $\mathcal{Q}^{\perp}$, then the shape operator A commutes with the structure tensor field $\phi$.

Proof. Applying $\xi=\xi_{1}$ into right hand side (resp., left hand side) of (ㄷ-1), we get

$$
\begin{aligned}
& 2 R_{\xi} \phi A X=-A \phi X+2 \alpha A^{2} \phi X-2 \eta_{3}(X) A \xi_{2}+2 \eta_{2}(X) A \xi_{3}-A \phi_{1} X, \\
& 2 A R_{\xi} \phi X=-\phi A X+2 \alpha A \phi A X-2 \eta_{3}(A X) \xi_{2}+2 \eta_{2}(A X) \xi_{3}-\phi_{1} A X .
\end{aligned}
$$

Combining (3.6) and (3.7), the above equations become

$$
\begin{aligned}
& R_{\xi} \phi A X=-A \phi X+\alpha A^{2} \phi X, \\
& A R_{\xi} \phi X=-\phi A X+\alpha A \phi A X .
\end{aligned}
$$

Hence, (C-1) is equivalent to

$$
A \phi-\phi A=\alpha A(A \phi-\phi A)
$$

Taking the symmetric part of (3.10), we have

$$
A \phi-\phi A=\alpha(A \phi-\phi A) A \text {. }
$$

From this, we can divide into the following three cases:

First, let us consider an open subset $\mathfrak{U}=\{p \in M \mid \alpha(p) \neq 0\}$ of $M$. Naturally we can apply (3.10) and (3.11) on the open subset $\mathfrak{U}$.

$$
(A \phi-\phi A) A X=A(A \phi-\phi A) X .
$$

Since the shape operator $A$ and the tensor $A \phi-\phi A$ are both symmetric operators and commute with each other, there exists a common orthonormal basis $\left\{E_{i}\right\}_{i=1, \ldots, 4 m-1}$ which gives a simultaneous diagonalization. Specifically, we have

$$
\begin{gathered}
A E_{i}=\lambda_{i} E_{i}, \\
(A \phi-\phi A) E_{i}=\beta_{i} E_{i},
\end{gathered}
$$

where $\lambda_{i}$ and $\beta_{i}$ are scalars for all $i=1,2, \ldots, 4 m-1$.

Taking the inner product with $E_{i}$ into (3.13), we have

$$
\beta_{i} g\left(E_{i}, E_{i}\right)=g\left((A \phi-\phi A) E_{i}, E_{i}\right)=2 \lambda_{i} g\left(\phi E_{i}, E_{i}\right)=0 .
$$


Since $g\left(E_{i}, E_{i}\right)=1, \beta_{i}=0$ for all $i=1,2, \ldots, 4 m-1$. Hence $A \phi X=\phi A X$ for any tangent vector field $X$ on $\mathfrak{U}$.

Next, if $p \in \operatorname{Int}(M-\mathfrak{U})$, then $\alpha(p)=0$. From this, the equation (3.11) gives $(A \phi-\phi A) X(p)=0$.

Finally, let us assume that $p \in \partial(M-\mathfrak{U})$, where $\partial(M-\mathfrak{U})$ is the boundary of $M-\mathfrak{U}$. Then there exists a subsequence $\left\{p_{n}\right\} \subset \mathfrak{U}$ such that $p_{n} \rightarrow p$. Since $(A \phi-\phi A) X\left(p_{n}\right)=0$ on the open subset $\mathfrak{U}$ in $M$, by the continuity we also get $(A \phi-\phi A) X(p)=0$.

Summing up these observations, it is natural that the shape operator $A$ commutes with the structure tensor field $\phi$ under our assumption.

By [11 we assert $M$ with the assumptions given in lemma 3.2 is locally congruent to one of the following hypersurfaces:

$\left(\mathcal{T}_{A}\right)$ a tube over a totally geodesic $S U_{2, m-1} / S\left(U_{2} \cdot U_{m-1}\right)$ in $S U_{2, m} / S\left(U_{2} \cdot U_{m}\right)$ or,

$\left(\mathcal{H}_{A}\right)$ a horosphere in $S U_{2, m} / S\left(U_{2} \cdot U_{m}\right)$ whose center at infinity is singular and of type $J X \in \mathfrak{J} X$.

In a paper due to [1], Suh gave some information related to the shape operator $A$ of $\mathcal{T}_{A}$ and $\mathcal{H}_{A}$ as follows:

Proposition A. Let $M$ be a connected real hypersurface in complex hyperbolic twoplane Grassamannian $S U_{2, m} / S\left(U_{2} U_{m}\right), m \geq 3$. Assume that the maximal complex subbundle $\mathcal{C}$ of $T M$ and the maximal quaternionic subbundle $\mathcal{Q}$ of TM are both invariant under the shape operator of $M$. If $J N \in \mathfrak{J} N$, then one of the following statements holds:

$\left(\mathcal{T}_{A}\right) M$ has exactly four distinct constant principal curvatures

$$
\alpha=2 \operatorname{coth}(2 r), \beta=\operatorname{coth}(r), \lambda_{1}=\tanh (r), \lambda_{2}=0,
$$

and the corresponding principal curvature spaces are

$$
T_{\alpha}=T M \ominus \mathcal{C}, T_{\beta}=\mathcal{C} \ominus \mathcal{Q}, T_{\lambda_{1}}=E_{-1}, T_{\lambda_{2}}=E_{+1} .
$$

The principal curvature spaces $T_{\lambda_{1}}$ and $T_{\lambda_{2}}$ are complex (with respect to $J$ ) and totally complex (with respect to $\mathfrak{J}$ ).

$\left(\mathcal{H}_{A}\right) M$ has exactly three distinct constant principal curvatures

$$
\alpha=2, \beta=1, \lambda=0
$$

with corresponding principal curvature spaces

$$
T_{\alpha}=T M \ominus \mathcal{C}, T_{\beta}=(\mathcal{C} \ominus \mathcal{Q}) \oplus E_{-1}, T_{\lambda}=E_{+1} .
$$

Here, $E_{+1}$ and $E_{-1}$ are the eigenbundles of $\left.\phi \phi_{1}\right|_{\mathcal{Q}}$ with respect to the eigenvaleus +1 and -1 , respectively.

Since the symmetric tensor $A \phi-\phi A$ vanishes identically on $\mathcal{T}_{A}$ (resp. $\mathcal{H}_{A}$ ), it trivially satisfies (3.10). Hence we assert that $\mathcal{T}_{A}$ (resp., $\mathcal{H}_{A}$ ) in complex hyperbolic two-plane Grassmannians $S U_{2, m} / S\left(U_{2} \cdot U_{m}\right)$ has the our commuting condition (C-1) (see [11]).

Next, due to Lemma 3.1, let us suppose that $\xi \in \mathcal{Q}$ (i.e., $J N \perp \mathfrak{J} N$ ).

By virtue of the result in [13, we assert that a Hopf hypersurface $M$ in complex hyperbolic two-plane Grassmannians $S U_{2, m} / S\left(U_{2} \cdot U_{m}\right)$ satisfying the hypotheses in Theorem 1 is locally congruent to 
$\left(\mathcal{T}_{B}\right) M$ is an open part of a tube around a totally geodesic quaternionic hyperbolic space $\mathbb{H} H^{n}$ in $S U_{2,2 n} / S\left(U_{2} U_{2 n}\right), m=2 n$,

$\left(\mathcal{H}_{B}\right) M$ is an open part of a horosphere in $S U_{2, m} / S\left(U_{2} U_{m}\right)$ whose center at infinity is singular and of type $J N \perp \mathfrak{J} N$, or

$(\mathcal{E})$ The normal bundle $\nu M$ of $M$ consists of singular tangent vectors of type $J X \perp \mathfrak{J} X$,

when $\xi \in \mathcal{Q}$. Hereafter, the model spaces of $\mathcal{T}_{B}, \mathcal{H}_{B}$ or $\mathcal{E}$ is denoted by $M_{B}$. Let us check whether the shape operator $A$ of model spaces of $M_{B}$ satisfy our conditions, conversely. In order to do this, let us introduce the following proposition given by Suh 13 .

Proposition B. Let $M$ be a connected hypersurface in $S U_{2, m} / S\left(U_{2} U_{m}\right), m \geq 3$. Assume that the maximal complex subbundle $\mathcal{C}$ of $T M$ and the maximal quaternionic subbundle $\mathcal{Q}$ of $T M$ are both invariant under the shape operator of $M$. If $J N \perp \mathfrak{J} N$, then one of the following statements holds:

$\left(\mathcal{T}_{B}\right) M$ has five (four for $r=\sqrt{2} \tanh ^{-1}(1 / \sqrt{3})$ in which case $\alpha=\lambda_{2}$ ) distinct constant principal curvatures

$$
\begin{aligned}
\alpha & =\sqrt{2} \tanh (\sqrt{2} r), \beta=\sqrt{2} \operatorname{coth}(\sqrt{2} r), \gamma=0, \\
\lambda_{1} & =\frac{1}{\sqrt{2}} \tanh \left(\frac{1}{\sqrt{2}} r\right), \quad \lambda_{2}=\frac{1}{\sqrt{2}} \operatorname{coth}\left(\frac{1}{\sqrt{2}} r\right),
\end{aligned}
$$

and the corresponding principal curvature spaces are

$$
T_{\alpha}=T M \ominus \mathcal{C}, T_{\beta}=T M \ominus \mathcal{Q}, T_{\gamma}=J(T M \ominus \mathcal{Q})=J T_{\beta} .
$$

The principal curvature spaces $T_{\lambda_{1}}$ and $T_{\lambda_{2}}$ are invariant under $\mathfrak{J}$ and are mapped onto each other by $J$. In particular, the quaternionic dimension of $S U_{2, m} / S\left(U_{2} U_{m}\right)$ must be even.

$\left(\mathcal{H}_{B}\right) M$ has exactly three distinct constant principal curvatures

$$
\alpha=\beta=\sqrt{2}, \gamma=0, \lambda=\frac{1}{\sqrt{2}}
$$

with corresponding principal curvature spaces

$$
T_{\alpha}=T M \ominus(\mathcal{C} \cap \mathcal{Q}), T_{\gamma}=J(T M \ominus \mathcal{Q}), T_{\lambda}=\mathcal{C} \cap \mathcal{Q} \cap J \mathcal{Q} .
$$

$(\mathcal{E}) M$ has at least four distinct principal curvatures, three of which are given by

$$
\alpha=\beta=\sqrt{2}, \gamma=0, \lambda=\frac{1}{\sqrt{2}}
$$

with corresponding principal curvature spaces

$$
T_{\alpha}=T M \ominus(\mathcal{C} \cap \mathcal{Q}), T_{\gamma}=J(T M \ominus \mathcal{Q}), T_{\lambda} \subset \mathcal{C} \cap \mathcal{Q} \cap J \mathcal{Q} .
$$

If $\mu$ is another (possibly nonconstant) principal curvature function, then $J T_{\mu} \subset T_{\lambda}$ and $\mathfrak{J} T_{\mu} \subset T_{\lambda}$. Thus, the corresponding multiplicities are

$$
m(\alpha)=4, \quad m(\gamma)=3, \quad m(\lambda), \quad m(\mu) .
$$

Let us assume that the structure Jacobi operator $R_{\xi}$ of $M_{B}$ satisfies the property (C-1). The tangent space of $M_{B}$ can be splitted into

$$
T M=T_{\alpha_{1}} \oplus T_{\alpha_{2}} \oplus T_{\alpha_{3}} \oplus T_{\alpha_{4}} \oplus T_{\alpha_{5}},
$$

where $T_{\alpha_{1}}=[\xi], T_{\alpha_{2}}=\operatorname{span}\left\{\xi_{1}, \xi_{2}, \xi_{3}\right\}, T_{\alpha_{3}}=\operatorname{span}\left\{\phi \xi_{1}, \phi \xi_{2}, \phi \xi_{3}\right\}$ and $T_{\alpha_{4}} \oplus T_{\alpha_{5}}$ is the orthogonal complement of $T_{\alpha_{1}} \oplus T_{\alpha_{2}} \oplus T_{\alpha_{3}}$ in $T M$. Since $\xi \in \mathcal{Q}$ and $\phi \phi_{\nu} \xi=$ 
$\phi^{2} \xi_{\nu}=-\xi_{\nu}$, we have $R_{\xi}\left(\phi \xi_{2}\right)=-2 \phi_{2} \xi$. From this and $\alpha_{3}=0$ for all $M_{B}$, our commuting condition (C-1) becomes

$$
R_{\xi} \phi A \xi_{2}-A R_{\xi} \phi \xi_{2}=-2 \alpha_{2} \phi \xi_{2} .
$$

It implies that the eigenvalue $\alpha_{2}$ vanishes, since $\phi \xi_{2}$ is a unit tangent vector field. But in Proposition B, for $\mathcal{T}_{B}$ (resp. $\mathcal{H}_{B}$ or $\mathcal{E}$ ) we see that the eigenvalue $\alpha_{2}=\beta=$ $\sqrt{2} \operatorname{coth}(\sqrt{2} r)\left(\right.$ resp. $\left.\alpha_{2}=\alpha=\frac{1}{\sqrt{2}}\right)$ is non-vanishing. This gives us a contradiction.

\section{Proof of Theorem 2}

In this section, by using geometric quantities in [3, 4, [5, 13, 14, and [15, we give a complete proof of Theorem 2. To prove it, we assume that $M$ is a Hopf hypersurface in $S U_{2, m} / S\left(U_{2} \cdot U_{m}\right)$ with commuting structure Jacobi operator and Ricci tensor, that is,

$$
\left(R_{\xi} \phi\right) S X=S\left(R_{\xi} \phi\right) X
$$

From the definition of the Ricci tensor and the fundamental formulas in 15, Section 2], the Ricci tensor $S$ of $M$ in $S U_{2, m} / S\left(U_{2} \cdot U_{m}\right)$ is given by

$$
\begin{aligned}
2 S X= & -(4 m+7) X+3 \eta(X) \xi+2 h A X-2 A^{2} X \\
& +\sum_{\nu=1}^{3}\left\{3 \eta_{\nu}(X) \xi_{\nu}-\eta_{\nu}(\xi) \phi_{\nu} \phi X+\eta_{\nu}(\phi X) \phi_{\nu} \xi+\eta(X) \eta_{\nu}(\xi) \xi_{\nu}\right\},
\end{aligned}
$$

where $h$ denotes the trace of the shape operator $A$.

Using equations (C-2) and (4.1), we prove that the Reeb vector field $\xi$ of $M$ belongs to either the distribution $\mathcal{Q}$ or the distribution $\mathcal{Q}^{\perp}$.

Lemma 4.1. Let $M$ be a Hopf hypersurface in $S U_{2, m} / S\left(U_{2} \cdot U_{m}\right), m \geq 3$, with (C-2). If the principal curvature $\alpha=g(A \xi, \xi)$ is constant along the direction of $\xi$, then $\xi$ belongs to either the distribution $\mathcal{Q}$ or the distribution $\mathcal{Q}^{\perp}$.

Proof. In order to prove this lemma, for some unit vectors $X_{0} \in \mathcal{Q}, \xi_{1} \in \mathcal{Q}^{\perp}$, we put

$$
\xi=\eta\left(X_{0}\right) X_{0}+\eta\left(\xi_{1}\right) \xi_{1},
$$

where $\eta\left(X_{0}\right) \eta\left(\xi_{1}\right) \neq 0$ is the assumption we will disprove in this proof by contradiction.

Let $\mathfrak{U}=\{p \in M \mid \alpha(p) \neq 0\}$ be the open subset of $M$. From now on, we discuss our arguments on $\mathfrak{U}$.

By virtue of Lemma 2.1, $\xi \alpha=0$ gives $A X_{0}=\alpha X_{0}$ and $A \xi_{1}=\alpha \xi_{1}$. From (4.1), we have

$$
\left\{\begin{array}{l}
S \phi X_{0}=\kappa \phi X_{0} \\
S X_{0}=\left(-2 m-4+h \alpha-\alpha^{2}\right) X_{0}+2 \eta\left(X_{0}\right) \xi \\
S \xi_{1}=\left(-2 m-2+h \alpha-\alpha^{2}\right) \xi_{1}+2 \eta_{1}(\xi) \xi \\
S \xi=\left(-2 m-2+h \alpha-\alpha^{2}\right) \xi+2 \eta_{1}(\xi) \xi_{1},
\end{array}\right.
$$

where $\kappa:=-2 m-4+h \sigma-\sigma^{2}$ and $\sigma=\frac{\alpha^{2}-2 \eta^{2}\left(X_{0}\right)}{\alpha}$ on $\mathfrak{U}$. 
Put $X=\phi X_{0}$ into (

$$
\kappa R_{\xi}\left(X_{0}\right)=S R_{\xi}\left(X_{0}\right) .
$$

Taking the inner product of (4.3) with $\xi$ and using (3.4) and (4.2), we have $-2 \alpha^{2} \eta^{2}\left(\xi_{1}\right) \eta\left(X_{0}\right)=0$. It implies that $\mathfrak{U}=\emptyset$. Thus it must be $p \in M-\mathfrak{U}$. The set $M-\mathfrak{U}=\operatorname{Int}(M-\mathfrak{U}) \cup \partial(M-\mathfrak{U})$, where Int (resp., $\partial$ ) denotes the interior (resp., the boundary) of $M-\mathfrak{U}$, we consider the following two cases:

- Case 1. $p \in \operatorname{Int}(M-\mathfrak{U})$

If $p \in \operatorname{Int}(M-\mathfrak{U})$, then $\alpha=0$. Our lemma was proved on $\operatorname{Int}(M-\mathfrak{U})$ by the equation (2.11) and (*).

- Case 2. $p \in \partial(M-\mathfrak{U})$

Since $p \in \partial(M-\mathfrak{U})$, there exists a sequence of points $p_{n} \in \mathfrak{U}$ such that $p_{n} \rightarrow p$ with $\alpha(p)=0$ and $\alpha\left(p_{n}\right) \neq 0$. Such a sequence will have an infinite subsequence where $\eta\left(\xi_{1}\right)=0$ (in which case $\xi \in \mathcal{Q}$ at $p$, by the continuity) or an infinite subsequence where $\eta\left(X_{0}\right)=0$ (in which case $\xi \in \mathcal{Q}^{\perp}$ at $p$ ). Accordingly, we get a complete proof of the Lemma.

Now, we shall divide our consideration into two cases that $\xi$ belongs to either the distribution $\mathcal{Q}$ or the distribution $\mathcal{Q}^{\perp}$, respectively. Let us consider the case $\xi \in \mathcal{Q}^{\perp}$. We may put $\xi=\xi_{1} \in \mathcal{Q}^{\perp}$ for the sake of convenience. Then, (4.1) is simplified:

$$
\begin{aligned}
2 S X= & -(4 m+7) X+7 \eta(X) \xi+2 \eta_{2}(X) \xi_{2} \\
& +2 \eta_{3}(X) \xi_{3}-\phi_{1} \phi X+2 h A X-2 A^{2} X .
\end{aligned}
$$

By replacing $X$ as $A X$ into (4.4) and using (3.8), we obtain

$$
2 S A X=-(4 m+6) A X+6 \alpha \eta(X) \xi+2 h A^{2} X-2 A^{3} X
$$

Applying the shape operator $A$ to (4.4) and using (3.9), we get

$$
2 A S X=-(4 m+6) A X+6 \alpha \eta(X) \xi+2 h A^{2} X-2 A^{3} X .
$$

From (4.5) and (4.6), we see that the Ricci tensor $S$ commutes with the shape operator $A$, that is,

$$
S A=A S .
$$

On the other hand, the equations (3.6) and (4.4) give us

$$
\begin{aligned}
2 \eta_{3}(S X) \xi_{2}-2 \eta_{2}(S X) \xi_{3}+\phi_{1} S X-\phi S X \\
\quad=(2 m+4)\left\{2 \eta_{3}(X) \xi_{2}-2 \eta_{2}(X) \xi_{3}+\phi X-\phi_{1} X\right\} \\
\quad:=\operatorname{Rem}(X) .
\end{aligned}
$$

Taking the symmetric part of (4.8), we obtain

$$
2 \eta_{3}(X) S \xi_{2}-2 \eta_{2}(X) S \xi_{3}+S \phi_{1} X-S \phi X=\operatorname{Rem}(X) .
$$

Lemma 4.2. Let $M$ be a Hopf hypersurface in $S U_{2, m} / S\left(U_{2} \cdot U_{m}\right)$ with (C-2). If $\xi \in \mathcal{Q}^{\perp}$, then $S \phi=\phi S$. 
Proof. By virtue of equation (4.8) and (4.9), we obtain the left and right sides of (C-2), respectively, as follows:

$$
\begin{aligned}
2 R_{\xi} \phi S X & =-\phi S X+2 \alpha A \phi S X-2 \eta_{3}(S X) \xi_{2}+2 \eta_{2}(S X) \xi_{3}-\phi_{1} S X \\
& =-2 \phi S X+2 \alpha A \phi S X-\operatorname{Rem}(X),
\end{aligned}
$$

and

$$
\begin{aligned}
2 S R_{\xi} \phi X & =-S \phi X+2 \alpha S A \phi X-2 \eta_{3}(X) S \xi_{2}+2 \eta_{2}(X) S \xi_{3}-S \phi_{1} X \\
& =-2 S \phi X+2 \alpha S A \phi X-\operatorname{Rem}(X) .
\end{aligned}
$$

That is,

$$
R_{\xi} \phi S X=-\phi S X+\alpha A \phi S X-\frac{1}{2} \operatorname{Rem}(X)
$$

and

$$
S R_{\xi} \phi X=-S \phi X+\alpha S A \phi X-\frac{1}{2} \operatorname{Rem}(X) .
$$

From these two equations, the condition (C-2) is equivalent to

$$
\begin{aligned}
(S \phi-\phi S) X & =\alpha(S A \phi-A \phi S) X \\
& =\alpha A(S \phi-\phi S) X,
\end{aligned}
$$

by virtue of our assertion that the shape operator $A$ commutes the Ricci tensor $S$ with each other given in (4.7).

Taking the symmetric part of (4.12), we have

$$
(S \phi-\phi S) X=\alpha(S \phi-\phi S) A X
$$

for all tangent vector fields $X$ on $M$.

From (4.12) and (4.13), we know

$$
\alpha A(S \phi-\phi S)=\alpha(S \phi-\phi S) A .
$$

Let $\mathfrak{U}=\{p \in M \mid \alpha(p) \neq 0\}$ be an open subset of $M$. Then (4.14) implies the shape operator $A$ and the symmetric tensor $S \phi-\phi S$ commute with each other on $\mathfrak{U}$. Hence they are simultaneous diagonalizable, there exists a common orthonormal basis $\left\{E_{1}, E_{2}, \ldots, E_{4 m-1}\right\}$ such that the shape operator $A$ and the tensor $S \phi-\phi S$ both can be diagonalizable. In other words,

$$
\begin{gathered}
A E_{i}=\lambda_{i} E_{i}, \\
(S \phi-\phi S) E_{i}=\beta_{i} E_{i},
\end{gathered}
$$

where $\lambda_{i}$ and $\beta_{i}$ are scalars for all $i=1,2, \ldots 4 m-1$.

Combining equations in (4.1), we get

$$
S \phi X-\phi S X=h A \phi X-A^{2} \phi X-h \phi A X+\phi A^{2} X .
$$

Using (4.15), (4.16) and (4.17), we obtain

$$
(S \phi-\phi S) E_{i}=h A \phi E_{i}-A^{2} \phi E_{i}-h \lambda_{i} \phi E_{i}+\lambda_{i}^{2} \phi E_{i} .
$$

Taking the inner product with $E_{i}$ into (4.18), we have

$$
\beta_{i} g\left(E_{i}, E_{i}\right)=h \lambda_{i} g\left(\phi E_{i}, E_{i}\right)-\lambda_{i}^{2} g\left(\phi E_{i}, E_{i}\right)-h \lambda_{i} g\left(\phi E_{i}, E_{i}\right)+\lambda_{i}^{2} g\left(\phi E_{i}, E_{i}\right)=0 .
$$

Since $g\left(E_{i}, E_{i}\right)=1$, we get $\beta_{i}=0$ for all $i=1,2, \ldots, 4 m-1$. This is equivalent to $(S \phi-\phi S) E_{i}=0$ for all $i=1,2, \ldots, 4 m-1$. It follows that $S \phi X=\phi S X$ for any 
tangent vector field $X$ on $\mathfrak{U}$. Next, if $p \in \operatorname{Int}(M-\mathfrak{U})$, then we see that $\alpha(p)=0$. From this, the equation (4.12) gives $(S \phi-\phi S)$ vanishes identically on $\operatorname{Int}(M-\mathfrak{U})$.

Finally, let us assume that $p \in \partial(M-\mathfrak{U})$, where $\partial(M-\mathfrak{U})$ is the boundary of $M-\mathfrak{U}$. Then there exists a subsequence $\left\{p_{n}\right\} \subset \mathfrak{U}$ such that $p_{n} \rightarrow p$. Since $(S \phi-\phi S) X\left(p_{n}\right)=0$ on the open subset $\mathfrak{U}$ in $M$, by the continuity we also get $(S \phi-\phi S) X(p)=0$.

By virtue of the result given by Suh in 14, we assert that if $\xi \in \mathcal{Q}^{\perp}$, then a Hopf hypersurface $M$ in $S U_{2, m} / S\left(U_{2} \cdot U_{m}\right)$ with (C-2) is locally congruent to one of the following hypersurfaces:

$\left(\mathcal{T}_{A}\right)$ a tube over a totally geodesic $S U_{2, m-1} / S\left(U_{2} \cdot U_{m-1}\right)$ in $S U_{2, m} / S\left(U_{2} \cdot U_{m}\right)$ or,

$\left(\mathcal{H}_{A}\right)$ a horosphere in $S U_{2, m} / S\left(U_{2} \cdot U_{m}\right)$ whose center at infinity is singular and of type $J X \in \mathfrak{J} X$.

Moreover, when $\xi \in \mathcal{Q}^{\perp}$, (C-2) is equivalent to (4.12). Since the symmetric tensor $(S \phi-\phi S)$ vanishes identically on $\mathcal{T}_{A}$ (resp. $\left.\mathcal{H}_{A}\right)$, it trivially satisfies (4.12). Hence we assert that $\mathcal{T}_{A}$ (resp., $\mathcal{H}_{A}$ ) in complex hyperbolic two-plane Grassmannians $S U_{2, m} / S\left(U_{2} \cdot U_{m}\right)$ has the our commuting condition (C-2) (see [14]).

When $\xi \in \mathcal{Q}$, a Hopf hypersurface $M$ in $S U_{2, m} / S\left(U_{2} \cdot U_{m}\right)$ with (C-2) is locally congruent to a hypersurface of $M_{B}$ by [13. From now on, let us show whether model spaces of $M_{B}$ satisfy the condition ( $(\mathrm{C}-2)$ or not. Then the tangent space of $M_{B}$ can be splitted into

$$
T M_{B}=T_{\alpha_{1}} \oplus T_{\alpha_{2}} \oplus T_{\alpha_{3}} \oplus T_{\alpha_{4}} \oplus T_{\alpha_{5}} .
$$

where $T_{\alpha_{1}}=[\xi], T_{\alpha_{2}}=\operatorname{span}\left\{\xi_{1}, \xi_{2}, \xi_{3}\right\}, T_{\alpha_{3}}=\operatorname{span}\left\{\phi \xi_{1}, \phi \xi_{2}, \phi \xi_{3}\right\}$ and $T_{\alpha_{4}} \oplus T_{\alpha_{5}}$ is the orthogonal complement of $T_{\alpha_{1}} \oplus T_{\alpha_{2}} \oplus T_{\alpha_{3}}$ in $T M$ such that $J T_{\alpha_{5}} \subset T_{\alpha_{4}}$ (see [14]).

On $T_{p} M_{B}, p \in M_{B}$, the equations (4.1) and (3.1) are reduced to the following equations, respectively:

$$
\begin{aligned}
2 S X=- & (4 m+7) X+3 \eta(X) \xi+2 h A X-2 A^{2} X \\
& +\sum_{\nu=1}^{3}\left\{3 \eta_{\nu}(X) \xi_{\nu}+\eta\left(\phi_{\nu} X\right) \phi_{\nu} \xi\right\}, \\
2 R_{\xi}(X)=- & X+\eta(X) \xi+2 \alpha A X-2 \alpha^{2} \eta(X) \xi \\
& +\sum_{\nu=1}^{3}\left\{\eta_{\nu}(X) \xi_{\nu}+3 \eta_{\nu}(\phi X) \phi_{\nu} \xi\right\} .
\end{aligned}
$$

From [14, Proposition 5.1], we obtain the following

$$
S X= \begin{cases}\left(-2 m-2+h \alpha_{1}-\alpha_{1}^{2}\right) \xi & \text { if } X=\xi \in T_{\alpha_{1}} \\ \left(-2 m-2+h \alpha_{2}-\alpha_{2}^{2}\right) \xi_{\ell} & \text { if } X=\xi_{\ell} \in T_{\alpha_{2}} \\ (-2 m-4) \phi \xi_{\ell} & \text { if } X=\phi \xi_{\ell} \in T_{\alpha_{3}} \\ \left(-2 m-\frac{7}{2}+h \alpha_{4}-\lambda_{4}^{2}\right) X & \text { if } X \in T_{\alpha_{4}} \\ \left(-2 m-\frac{7}{2}+h \alpha_{5}-\alpha_{5}^{2}\right) X & \text { if } X \in T_{\alpha_{5}}\end{cases}
$$




$$
R_{\xi}(X)= \begin{cases}0 & \text { if } X=\xi \in T_{\alpha_{1}} \\ \alpha_{1} \alpha_{2} \xi_{\ell} & \text { if } X=\xi_{\ell} \in T_{\alpha_{2}} \\ \left(-2+\alpha_{1} \alpha_{3}\right) \phi \xi_{\ell} & \text { if } X=\phi \xi_{\ell} \in T_{\alpha_{3}} \\ \left(-\frac{1}{2}+\alpha_{1} \alpha_{4}\right) X & \text { if } X \in T_{\alpha_{4}} \\ \left(-\frac{1}{2}+\alpha_{1} \alpha_{5}\right) X & \text { if } X \in T_{\alpha_{5}} .\end{cases}
$$

In order to check whether $\mathcal{T}_{B}, \mathcal{H}_{B}$ or $\mathcal{E}$ model spaces satisfy the (C-2) or not, we should verify the following equations vanishes for all cases.

$$
G(X):=\left(R_{\xi} \phi\right) S X-S\left(R_{\xi} \phi\right) X .
$$

Putting $X=\xi_{1} \in T_{\alpha_{3}}$ into (4.21), we have $G\left(\xi_{1}\right)=-2\left(2+\alpha_{2} h-\alpha_{2}^{2}\right) \phi \xi_{1}$ which derives

$$
2+\alpha_{2} h-\alpha_{2}^{2}=0
$$

- Case 1. Tube $\mathcal{T}_{B}$

In this case, we get $\alpha_{1}=\alpha, \alpha_{2}=\beta, \alpha_{3}=\gamma=0, \alpha_{4}=\lambda$ and $\alpha_{5}=\mu$.

By calculation, we have $\lambda+\mu=\beta$ on $\mathcal{T}_{B}$. Thus we obtain $h=\alpha+3 \beta+$ $(4 n-4)(\lambda+\mu)=\alpha+(2 m-1) \beta$. Then (4.22) is $4+2(m-1) \beta^{2}>0$, which is a contradiction.

- Case 2. Horoshere $\mathcal{H}_{B}$

On $\mathcal{H}_{B}, \alpha_{1}=\sqrt{2}, \alpha_{2}=\sqrt{2}, \alpha_{3}=\gamma=0, \alpha_{4}=\frac{1}{\sqrt{2}}$ and $\alpha_{5}=\frac{1}{\sqrt{2}}$. Thus (4.22) gives $h=0$. Since $h=\alpha_{1}+3 \alpha_{2}+3 \alpha_{3}+(4 n-4)\left(\alpha_{4}+\alpha_{5}\right)$, we have $2 \sqrt{2} m=0$ which is a contradiction.

- Case 3. Exceptional case $\mathcal{E}$

For $X \in T_{\alpha_{5}} \subset T_{\mathcal{E}}, G(X)=-\frac{1}{2}\left(\alpha_{5}-\alpha_{4}\right)\left(\alpha_{5}+\alpha_{4}\right) \phi X$. On $T_{\mathcal{E}}$ we have $\alpha_{1}=$ $\alpha=\sqrt{2}, \alpha_{4}=\lambda=\frac{1}{\sqrt{2}}$ and $\alpha_{5}=\mu= \pm \frac{1}{\sqrt{2}}$. Because $\mu \neq \lambda$, it should be $\mu=-\frac{1}{\sqrt{2}}$. Moreover, since $J T_{\mu} \subset T_{\lambda}$ and $\mathfrak{J} T_{\mu} \subset T_{\lambda}$, we see that the corresponding multiplicities of the eigenvalues $\lambda$ and $\mu$ satisfy $m(\lambda) \geq m(\mu)$. Since $m(\alpha)=4$, $m(\gamma)=3$ and $m(\lambda)+m(\mu)=4 m-8$ on $\mathcal{E}$, the trace of the shape operator $A$ denoted by $h$ becomes $h=4 \alpha+3 \gamma+m(\lambda) \lambda+m(\mu) \mu=4 \sqrt{2}+\frac{1}{\sqrt{2}}(m(\lambda)-m(\mu))$, which makes a contradiction. In fact, since we obtained $h=0$ on $T_{\gamma} \in T \mathcal{E}$, it yields $(m(\lambda)-m(\mu))=-8<0$. Thus, this case does not occur.

This shows that hypersurfaces of $\mathcal{T}_{B}, \mathcal{H}_{B}$ or $\mathcal{E}$ cannot satisfy the condition (C-2), and therefore in the situation of Theorem 2, the case $X \in \mathcal{Q}$ cannot occur. This completes the proof of Theorem 2.

\section{REFERENCES}

[1] I. Jeong, J. D. Pérez and Y. J. Suh, Recurrent Jacobi operator of real hypersurfaces in complex two-plane Grassmannians, Bull. Korean Math. Soc. 50(2) (2013), 525-536.

[2] H. Lee, Y.S. Choi and C. Woo, Hopf hypersurfaces in complex two-plane Grassmannians with Reeb parallel shape operator, Bull. Malaysian Math. Soc. 38 (2015), 617-634.

[3] H. Lee, Y.J. Suh and C. Woo, Real hypersurfaces with commuting Jacobi operators in complex two-plane Grassmannians, Houston J. Math. 40 (2014), no. 3, 751-766. 
[4] H. Lee, Y.J. Suh and C. Woo, Reeb parallel Ricci tensor for homogeneous real hypersurfaces in complex hyperbolic two-plane Grassmannians, Math. Nachr. 288 (2015), 1-12.

[5] E. Pak, Y. J. Suh and C. Woo, Restricted Ricci conditions for real hypersurfaces in complex two-plane Grassmannians, Houston J. Math. 41 (2015) no. 3 767-783.

[6] J.D. Pérez, C. Machado, I. Jeong and Y.J. Suh, Real hypersurfaces in complex two-plane Grassmannians with $\mathfrak{D}^{\perp}$-parallel structure Jacobi operator, Inter. J. Math. 22 (2011), no. 5, 655-673.

[7] J.D. Pérez, C. Machado and Y.J. Suh, Commuting structure Jacobi operator for real hypersurfaces in complex two-plane Grassmannians, Acta Math. Sin. 31 (2015) no. 1, 111-122.

[8] J.D. Pérez and Y.J. Suh, The Ricci tensor of real hypersurfaces in complex two-plane Grassmannians, J. Korean Math. Soc. 44 (2007), 211-235.

[9] J.D. Pérez, Y.J. Suh and Y. Watanabe, Generalized Einstein real hypersurfaces in complex two-plane Grassmannians, J. Geom. Phys. 60 (2010), 1806-1818.

[10] J.D. Pérez, Y.J. Suh and C. Woo, Real Hypersurfaces in complex hyperbolic two-plane Grassmannians with commuting shape operator, Open Math. 13 (2015) 493-501.

[11] Y.J. Suh, Hypersurfaces with isometric Reeb flow in complex hyperbolic two-plane Grassmannians, Adv. Appl. Math. 50 (2013), 645-659.

[12] Y.J. Suh, Real hypersurfaces in complex two-plane Grassmannians with harmonic curvature, J. Math. Pures Appl. 100 (2013), 16-33.

[13] Y.J. Suh, Real hypersurfaces in complex hyperbolic two-plane Grassmannians with Reeb vector field, Adv. Appl. Math. 55 (2014), 131-145.

[14] Y.J. Suh, Real hypersurfaces in complex hyperbolic two-plane Grassmannians with commuting Ricci tensor, Inter. J. Math. World Sci. Publ., 26 (2015), 1550008 (26 pages).

[15] Y.J. Suh and C. Woo, Real hypersurfaces in complex hyperbolic two-plane Grassmannians with parallel Ricci tensor, Math. Nachr. 287 (2014), 1524-1529.

HyUnJin LEe

Research Institute of Real and Complex Manifold,

KyUngPOOK NATIONAL UNIVERSITY,

DAEGU 702-701, REPUBLIC OF KOREA

E-mail address: lhjibis@hanmail.net

Young Jin Suh

Department of Mathematics

and Research Institute of Real and Complex Manifold,

KYUNGPOOK NATIONAL UNIVERSITY,

DAEGU 702-701, REPUBLIC OF KOREA

E-mail address: yjsuh@knu.ac.kr

Changhwa Woo

Department of Mathematics,

KyUngPOOK National University,

DAEGU 702-701, REPUBLIC OF KOREA

E-mail address: legalgwch@naver.com 